\title{
Light collection from scattering media in a silicon photonics integrated circuit
}

\author{
Karel Van Acoleyen, Eva Ryckeboer, Katarzyna Komorowska and Roel Baets \\ Photonics Research Group, Department of Information Technology (INTEC) \\ Ghent University - imec, Belgium \\ Center for Nano- and Biophotonics (NB-Photonics), Ghent University, Belgium \\ Email: karel.vanacoleyen@intec.ugent.be
}

\begin{abstract}
We present a silicon photonics integrated circuit to efficiently couple scattered light into a single mode waveguide. By modulating the phase of $\mathrm{N}$ light-capturing elements, the collection efficiency can be increased by a factor $\mathrm{N}$.
\end{abstract}

\section{INTRODUCTION}

As photonic integration is advancing, more specialized applications can make use of its benefits. One of the key features of photonic integrated circuits is the interface between the outside world. In biological applications, one is often interested in collecting (back-)scattered light that has traveled through tissue to do e.g. Doppler velocimetry [1], Optical Coherence Tomography (OCT) [2] or Raman spectroscopy [3]. Efficient light collection is one of the key elements here. We propose to use an integrated probe on a silicon chip so that signal processing can happen immediately where the signal is picked up. This reduces the noise and other artifacts that are present in e.g. fiber probes [3]. An approach to integrate a probe for efficient collection of scattered light on the silicon oxynitride waveguide platform has been shown in [4]. A phase-retrieval integrated circuit is proposed here which consists of an array of light-capturing elements. The phase of these elements can be tuned so that everything adds up coherently into a single mode waveguide after which further processing can happen on-chip.

\section{DESIGN AND FABRICATION}

Light is coupled into the chip using grating couplers. These are optimized to couple light into the fundamental TE-like mode of an integrated waveguide. Depolarization effects are not taken into account here. Grating couplers have proven to be an efficient way to couple light between a fiber and an optical integrated circuit [5]. Here they are used as 'optical antennas' to couple between free-space and the integrated circuit. When one uses one large grating coupler to couple scattered light on-chip, the phase distortion due to the scattering will reduce the coupling efficiency dramatically when tapering to a single mode waveguide. By splitting up the large grating coupler in multiple small grating couplers and tuning their respective phases, the light can be more efficiently guided into a single mode waveguide.

The integrated probe was fabricated at imec using standard CMOS technology steps including deep-UV (Ultra Violet) lithography [6] on a SOI (Silicon-On-Insulator) wafer with

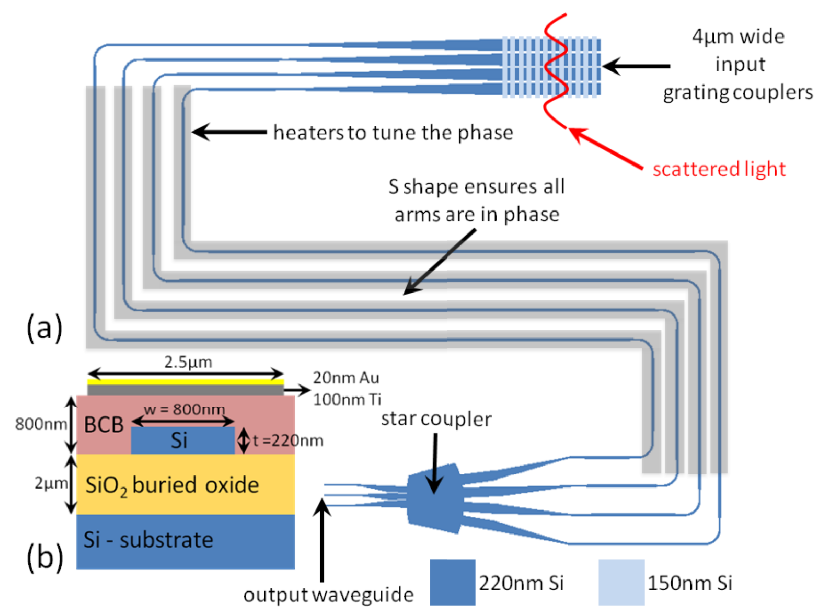

Fig. 1. (a) Schematic of the component, (b) Cross-sectional view of the heaters.

a $2 \mu \mathrm{m}$ buried oxide layer and a $220 \mathrm{~nm}$ top silicon layer. An etch step of $220 \mathrm{~nm}$ and $70 \mathrm{~nm}$ is then used to etch the waveguides, star coupler and grating couplers. Fig. 1(b) shows a cross-sectional view of the component, while Fig. 1(a) gives a complete overview. Light is coupled in through $N$ grating couplers, $4 \mu \mathrm{m}$ wide, spaced $5 \mu \mathrm{m}$. They taper back to a single mode waveguide of $450 \mathrm{~nm}$ wide. Afterwards light is guided through the $\mathrm{S}$-shape on which heaters are processed to tune the phases. In the S-shape, we taper back to an $800 \mathrm{~nm}$ wide waveguide to be more tolerant to fabrication errors. The heaters will tune the phases of the respective waveguides to optimize the coupling of the light in the star coupler to the output waveguide. This output waveguide tapers to a grating coupler optimized for coupling the light to an optical fiber. In future designs a detector can be integrated on the chip.

\section{THEORETICAL MODELING}

A biological medium can be modeled as a random Gaussian medium with a certain correlation length $l_{\text {coh }}$ which is a measure for the size of the particles. At first instance, we neglect the amplitude variations, and only phase fluctuations are taken into account using an electromagnetic approach. The phase fluctuation of a plane wave propagating through such a medium can then be calculated [7]. In Fig. 2 the phase fluctuation of a plane wave propagating through a $1 \mathrm{~mm}$ thick scattering medium with a fluctuation strength of $\sigma_{n}=0.02$ is 
plotted. For small scattering centers (and thus small $l_{c o h}$ ) the phase change is small and quickly varying, while for larger scattering centers, we get a larger effect but over a larger length scale. To effectively capture the phase, the different light-capturing elements should thus be small enough to follow these phase fluctuations. By adjusting the phases of the light

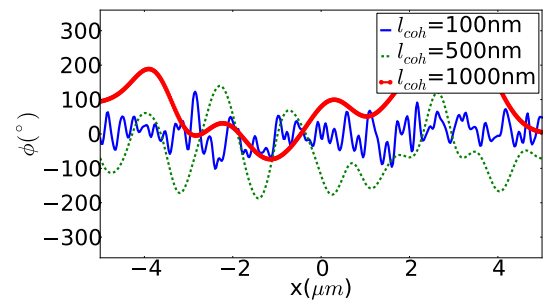

Fig. 2. Phase fluctuation of a plane wave after propagation through a $1 \mathrm{~mm}$ thick weakly scattering medium with different coherence lengths $l_{c o h}$ at a wavelength of $1550.0 \mathrm{~nm}$. The fluctuation strength $\sigma_{n}=0.02$

that is coupled in by the grating couplers, their contributions can be added up coherently in the star coupler. In Fig. 3, the scatter losses due to phase variations are shown with and without phase reconstruction for a 4 element array at $\lambda=1550 \mathrm{~nm}$. Fig. 3(a) shows us that there is a large fluctuation on the received power, but when phase compensation is used, the probability that the received signal is extremely low is reduced and the average received signal is increased with a factor of $6 \mathrm{~dB}(N=4)$ due to the coherent superposition of the different elements (Fig. 3(b)). When $l_{\text {coh }}$ is small, the phase fluctuation is small and rapid as seen in Fig. 2 and compensating for the phase has no effect when using $4 \mu \mathrm{m}$ wide grating couplers. Only for $l_{c o h}>500 \mathrm{~nm}$, we can see a distinct effect.

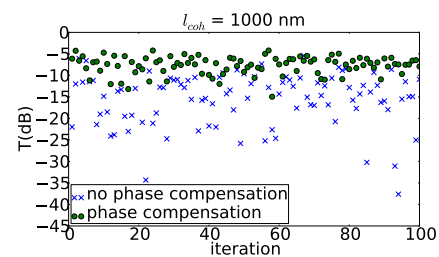

(a)

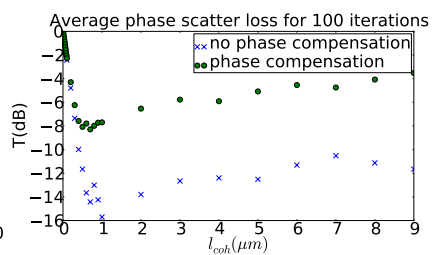

(b)
Fig. 3. (a) Phase scatter loss through a scattering medium $\left(l_{c o h}=1000 \mathrm{~nm}\right)$ of $1 \mathrm{~mm}$ thickness at $\lambda=1550 \mathrm{~nm}$ for different random realizations of the scattering medium. (b) Average phase scatter loss for 100 iterations as a function of coherence length $l_{c o h}$ of the scattering medium.

\section{Measurement Results}

Light from a tunable laser is guided through a polarization controller to a fiber collimator with a $1 / \mathrm{e}^{2}$ diameter of $7 \mathrm{~mm}$ through a $1 \mathrm{~mm}$ thick static scattering PDMS film (with unknown coherence length) and then focused with a microscope objective (NA=0.1) onto the structure with 4 grating couplers $(N=4)$. The light is then guided through the component and coupled to an optical fiber which is connected to an optical power meter. The heater currents are optimized to maximize the transmission using a simulated-annealing algorithm. This algorithm was chosen as it was proven to be robust against measurement fluctuations. An example of such an optimization run is shown in Fig. 4(a), where the red dashed line shows the starting point. In Fig. 4(b), this optimization was performed for different wavelengths where we can see a general increase of the transmission with a factor of around $4 \mathrm{~dB}$. The value of $4 \mathrm{~dB}$ represents only the gain over one sample of the ensemble of all possible phase distributions of the uncompensated measurement. In that respect the value of $4 \mathrm{~dB}$ is somewhat arbitrary but the experiment clearly demonstrates that phase compensation has a strong impact on the collected single-mode power.

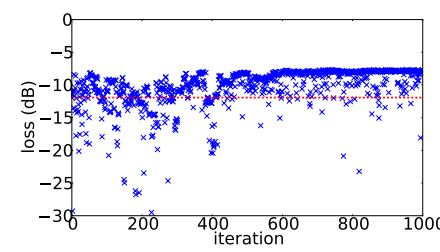

(a)

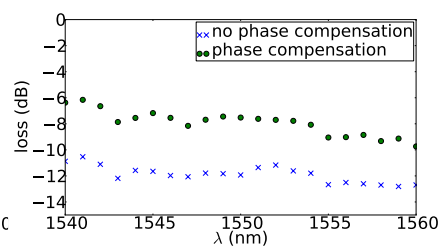

(b)
Fig. 4. (a) Measured optimization of the losses using simulated annealing, the red dashed line shows the starting point. (b) Measured losses before and after phase compensation for different wavelengths, an improvement of $4 \mathrm{~dB}$ can be seen.

\section{CONClusion}

Transmission through scattering media can be optimized using a phase reconstruction technique. The phases of the different light-capturing elements are optimized to coherently add up all the contributions into one single mode waveguide. An improvement of $4 \mathrm{~dB}$ was measured using 4 elements.

\section{ACKNOWLEDGMENT}

Karel Van Acoleyen acknowledges the Research Foundation - Flanders (FWO) for a research grant. This work was supported by the Methusalem project Smart Photonic ICs and the GlucoSens project of Ghent University and the IAP project Photonics@be. The author would like to thank Steven Verstuyft for the help in the processing of the heaters.

\section{REFERENCES}

[1] Y. Li, S. Meersman and R. Baets, "Realization of fiber based laser Doppler vibrometer with serrodyne frequency shifting," Applied Optics, to be published, ID 140343, 2011.

[2] G. Yurtsever, P. Dumon, W. Bogaerts, and R. Baets, "Integrated photonic circuit in silicon on insulator for Fourier domain optical coherence tomography," in Proceedings of SPIE-The International Society for Optical Engineering, vol. 7554, 2010.

[3] M. Balu, G. J. Liu, Z. P. Chen, B. J. Tromberg, and E. O. Potma, "Fiber delivered probe for efficient CARS imaging of tissues," Optics Express, vol. 18, no. 3, pp. 2380-2388, 2010.

[4] N. Ismail, F. Sun, K. Worhoff, A. Driessen, R. M. de Ridder, and M. Pollnau, "Excitation and Light Collection From Highly Scattering Media With Integrated Waveguides,' IEEE Photonics Technology Letters, vol. 23, no. 5, pp. 278-280, 2011.

[5] G. Roelkens, D. Vermeulen, D. Van Thourhout, R. Baets, S. Brision, P. Lyan, P. Gautier, and J. M. Fedeli, "High efficiency diffractive grating couplers for interfacing a single mode optical fiber with a nanophotonic silicon-on-insulator waveguide circuit," Applied Physics Letters, vol. 92, no. 13, p. 131101, 2008.

[6] ePIXfab, "The silicon photonics platform," http://www.epixfab.eu/, 2011.

[7] A. Ishimaru, "Wave Propagation and Scattering in Random Media," Academic Press, Inc., 1978. 